\title{
Rhythmicity in Primary Headache Disorders
}

\author{
Evgenia Koutsouki \\ Editor
}

Citation: EMJ Neurol. 2021;9[1]:21-23.

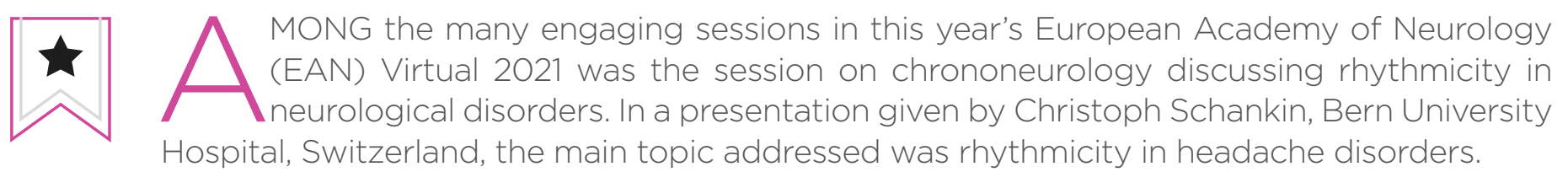

\section{CHRONOBIOLOGY AND RHYTHMICITY IN THE HUMAN BODY}

In his introductory remarks, Schankin explained the concept of chronobiology, which studies biological periodic phenomena that occur in cycles, giving rhythm to processes. Examples of such cycles that are adapted by environmental stimuli include the circadian rhythm, which is a 24-hour-long circle, and similarly, circaseptan cycles, which are cultural and based on the 7-day week, which includes weekends and work days. Another type of rhythm is the natural phenomenon of the moon phase lasting 28 days, which is important for the hormonal cycle in females, and finally the circannual rhythm, which refers to the year, the seasons, the length of the daytime, and temperatures.

Schankin explained that the circadian rhythm is present at a single-cell level and has a number of transcriptional products, among which are the CLOCK protein, Period 1 and 2, and Casein Kinase 1, which influence each other. The body organs formed by cells act as a single circadian unit or peripheral clock, each of which runs independently and is synchronised by a central pacemaker, the suprachiasmatic nucleus (SCN).
The SCN is a region in the hypothalamus that is calibrated by light via pituitary adenylate cyclase activating polypeptide (PACAP). Food, temperature, exercise, and circadian hormones, such as steroids and melatonin, are other calibrators of the SCN.

\section{RHYTHMICITY IN MIGRAINES}

When it comes to migraines, Schankin continued, there is clear evidence of a circadian rhythmicity, shown in that most migraine attacks start at noon or $1 \mathrm{pm}$. Further to this, a 2007 study on those affected by migraine distinguishing between early risers and late risers showed that early risers experience their migraine attacks earlier in the day, whereas, typically, late risers experience their migraine attacks later in the day. Interestingly, there is also evidence of circaseptan rhythmicity in migraines, as fewer migraines occur on Sundays than any other day. Finally, there is evidence of monthly migraine rhythmicity in females who are not on oral contraceptives, as it has been shown that they often experience their migraines on the first or second day of their menstrual period. 


\section{MIGRAINE TRIGGERS}

When discussing the underlying causes for this rhythmicity, Schankin emphasised the importance of the premonitory (early) phase of the migraine attack, which can start hours to days before the main headache phase. The hypothalamus, cortical, and subcortical areas are key areas implicated in this phase, as are neurotransmitters and neuropeptides such as noradrenaline, orexins, and dopamine. Discussing a study in which migraine attacks were triggered in patients using nitroglycerine infusions, and scanning the brain during the premonitory phase, Schankin explained that hypothalamic activation was observed. According to Schankin, one working theory is that the hypothalamus suppresses trigeminal cervical complex activity and any information that comes from the meninges is suppressed by this hypothalamic activity and not perceived as head pain. During a migraine attack, it is believed that there is a dysfunction in this hypothalamic suppressive activity and, as a result, patients perceive signals from the meninges as head pain. Similar mechanisms are involved in response to sound, light, and gastrointestinal function.

Although little is known about the involvement of neurotransmitters, Schankin presented an animal study that has shown differential involvement of orexins $A$ and $B$ in the trigeminal transmission mechanism.

It is not well-known which molecules might be involved in the migraine attack mechanism at a cellular level; however, Schankin highlighted casein kinase as an important player. People that carry a mutated form of this protein experience an early sleep phase syndrome and this is cosegregated with migraine with aura. An animal study by Brennan in 2013 found that there was a lower threshold for triggering migraine attacks in animals that carry this mutation compared to wild-type animals.

\section{RHYTHMICITY IN CLUSTER HEADACHES}

The rhythmicity of cluster headache has been extensively studied and it has now been shown that the majority of cluster headaches happen at night (1 or $2 \mathrm{am}$ ). When performing statistical analyses of the chrono-distribution of attacks to determine the oscillation period, a 2018 study found that the most prevalent oscillation period was the 24-hour period, followed by the 12-hour and 4.8-hour periods. Comparison of patients with episodic or chronic cluster headaches showed that these three periods were only present in patients with episodic cluster headaches, whereas the patients with chronic cluster headaches had a more diverse chrono-distribution of their headaches. In interpreting these results, Schankin explained that the group with chronic cluster headaches had a more disturbed chronobiology. In addition to this, cluster headaches appear to have a clear circannual rhythmicity. It is believed that cluster headaches occur in bouts when there are extremes in day length (summer and winter solstice). Other kinds of rhythmicities are observed in groups of patients who never get their cluster headaches in summer, or other patients who get their headaches on the winter or summer equinox.

\section{UNDERLYING CAUSES OF RHYTHMICITY}

Studies have looked into whether the causes for this rhythmicity could be genetic. Studies looking at the CLOCK gene and its variants found an association between some variants and the incidence of cluster headaches. Orexins are another factor that has been studied; reduced levels of orexin A were found in cerebrospinal fluid in cluster headaches. The orexin receptor has also been studied. A study from Italy and another from Germany found strong correlations between a mutation in the receptor and cluster headaches; however, other studies have not shown such a correlation. Schankin commented that the receptor might play an important role, however, the geographical area as well as the genetic background of the patient might also play a part. Finally, studies have shown that the PACAP gene variant is more prevalent in individuals that suffer from cluster headaches compared to controls.

\section{THE ROLE OF SLEEP AND THE HYPOTHALAMUS}

When it comes to studying the link between headache rhythmicity and sleep, Schankin explained that there seems to be a higher 


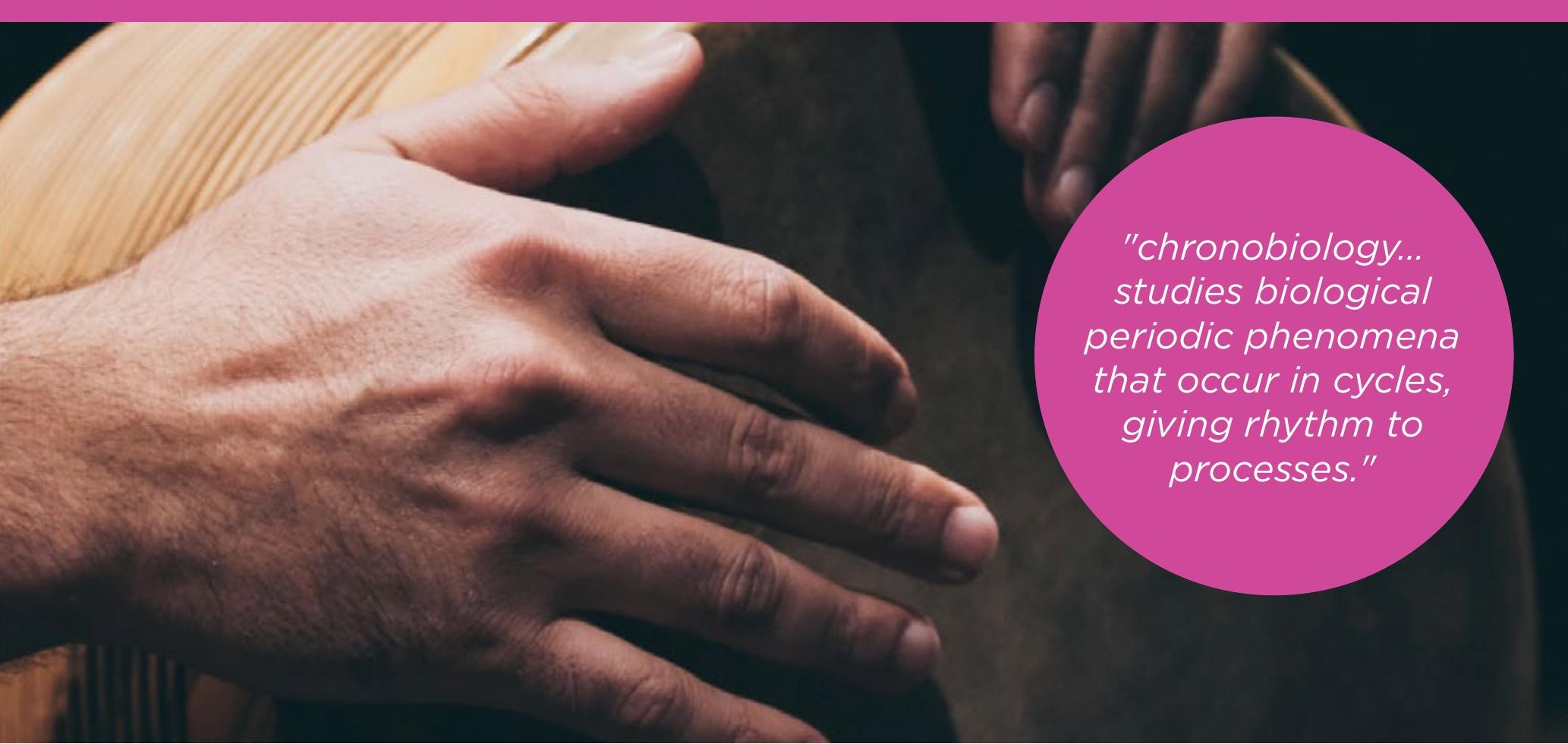

likelihood of having a cluster headache during rapid eye movement (REM) sleep, with 30\% of cluster headaches occurring at this period compared to $17 \%$ during non-REM sleep.

The hypothalamus also seems to play a role in cluster headaches as there appear to be be functional and structural alterations in the hypothalamic region and there are differences in melatonin excretion. Melatonin levels are increased in healthy patients at night, whereas with patients with cluster headaches have no such change in melatonin between the day and night.

\section{THE HYPNIC HEADACHE}

The hypnic headache, which occurs exclusively during sleep and causes awakening, is a type of headache likely to be REM-sleep-related as patients report it occurring during vivid dreams. Another possibility is that there is a chronobiological link as they usually occur at the same time in the night (mostly 2-3 am) and could involve the suprachiasmatic nucleus part of the hypothalamus and reduced melatonin secretion. However, a study by Holle in 2013 failed to find an association with REM sleep or any differences in melatonin secretion. What this study did show, however, was hypothalamic involvement in the hypnic headache, finding decreased grey matter density in the hypothalamus in patients with hypnic headache compared to controls.

\section{TREATMENT OPTIONS AND CONCLUDING REMARKS}

When putting this evidence together, Shankin explained that chronobiology plays a major role in primary headache syndromes and there is some evidence on the involvement of the hypothalamus and its major mediators. With regard to the role of sleep, it appears to be a twoway mechanism as sleep affects headaches and vice versa.

When it comes to treating rhythmicity in headaches, lithium is used for cluster and hypnic headache prophylaxis as it is an inhibitor of glycogen synthase kinase 3 B. Valproic acid is another therapy used as it shifts timing of Period2 protein. Randomised controlled trials in this topic from the 1990s have shown that melatonin is able to significantly reduce the number of cluster headaches and a recent study by Oberman confirmed that steroids were helpful in cluster headache prophylaxis.

With regard to orexin $A$ and $B$, however, no differences have been shown between placebo and an orexin inhibitor. Similarly, a study using PACAP antibody versus placebo showed no difference when used as migraine prophylaxis.

In his concluding remarks, Schankin said that medications altering rhythmicity could be helpful for treatment; however, a lot of evidence is still lacking. 